\title{
Entretien avec Tanika Sarkar. Écrire et enseigner l'histoire des femmes et des subalternes en Inde
}

Propos recueillis par courriel en septembre et décembre 2018 par Fabrice Bensimon

\section{Tanika Sarkar et Fabrice Bensimon}

Traducteur : Laurent Bury

\section{OpenEdition \\ Journals}

Édition électronique

URL : http://journals.openedition.org/rh19/5490

DOI : $10.4000 /$ rh19.5490

ISSN : $1777-5329$

Éditeur

La Société de 1848

Édition imprimée

Date de publication : 15 octobre 2018

Pagination : 113-125

ISSN : 1265-1354

Référence électronique

Tanika Sarkar et Fabrice Bensimon, «Entretien avec Tanika Sarkar. Écrire et enseigner l'histoire des femmes et des subalternes en Inde », Revue d'histoire du XIXe siècle [En ligne], 56 | 2018, mis en ligne le 15 octobre 2020, consulté le 05 janvier 2021. URL : http://journals.openedition.org/rh19/5490 ; DOI : https://doi.org/10.4000/rh19.5490 


\section{ENTRETIEN AVEC TANIKA SARKAR}

\section{Écrire et enseigner l'histoire des femmes et des subalternes en Inde}

Née en 1949 à Calcutta, dans une famille d'enseignants, Tanika Sarkar commence ses études universitaires au Bengale avant de rejoindre la Delhi University où elle soutient en 1981 sa thèse de doctorat. Celle-ci, intitulée Bengal 1928-1934: The Politics of Protest, s'inscrit dans le profond renouvellement que connait l'historiographie indienne à la fin des années 1970 et au début des années $1980^{1}$. Sous l'influence des écrits d'E. P. Thompson et de l'histoire radicale britannique, les postulats de l'historiographie nationaliste indienne sont remis en cause comme étant élitistes. L’attention est désormais portée à l'étude des «subalternes» et à leur culture politique. Tanika Sarkar enseigne ensuite aux St. Stephen's and Indraprastha Colleges à Delhi, avant de devenir professeure d'histoire contemporaine à la prestigieuse Jawaharlal Nehru University, de la même ville. Active dans le mouvement féministe et syndical, elle a beaucoup contribué à l'essor en Inde des études sur le genre. Elle est notamment membre du comité éditorial du Journal of Women's History et a publié, avec son mari l'historien Sumit Sarkar, une anthologie sur ces questions ${ }^{2}$.

RH19: Puisque cette interview paraîtra dans la Revue d'Histoire du $X I X^{e}$ siècle, nous voudrions d'abord aborder la fracture entre les $\mathrm{XIX}^{\mathrm{e}}$ et $\mathrm{XX}^{\mathrm{e}}$ siècles (après 1914) souvent utilisée dans l'historiographie du monde occidental. Cela semble beaucoup moins fréquent dans les ouvrages consacrés à l'histoire indienne. Comment l'expliquez-vous?

Tanika Sarkar : Vous avez tout à fait raison. Pour l'histoire de l'Occident, la Première Guerre mondiale représente la fin du long XIX ${ }^{\mathrm{e}}$ siècle et le début d'une ère nouvelle. En Inde, en revanche, ce conflit ne marque pas une 1987.

1. Tanika Sarkar, Bengal 1928-1934: The Politics of Protest, Delhi, Oxford University Press,

2. Tanika Sarkar and Sumit Sarkar (eds), Women and Social Reform in Modern India: A Reader, Bloomington, Indiana University Press, 2008. 
rupture aussi nette. Des travaux récents ont d'ailleurs révélé en quoi il avait affecté le cours de l'histoire indienne ${ }^{3}$. Néanmoins, il n'est pas perçu comme un tournant radical, essentiellement parce que les théâtres des opérations se situaient loin de l'Inde.

Le mode de périodisation standard que nous utilisons considère la période coloniale comme inaugurant la modernité indienne. Puisque l'autorité coloniale s'est fermement établie comme réalité pour le sous-continent au début $\mathrm{du} \mathrm{XIX}^{\mathrm{e}}$ siècle, pour se déployer lentement tout au long de ce siècle, c'est cet événement qui est pris comme véritable tournant. De plus, on estime en général que l'autorité coloniale a entièrement fait disparaître tous les liens avec le passé et a instauré une époque totalement différente et radicalement neuve. Certains historiens post-coloniaux, surtout dans les années 1990, affirmaient aussi que les Indiens avaient dès lors perdu la capacité de comprendre leurs propres traditions et leur propre histoire, tant ils étaient imprégnés du système de connaissance occidental.

Pourtant, une autre lecture caractéristique de l'histoire indienne moderne consiste à voir la période coloniale comme un tout, une unité historique sans solution de continuité, sans qu'il faille accorder une attention particulière aux ruptures et aux évolutions importantes qui se sont produites durant toute cette période. Même la phase post-indépendance est perçue comme un prolongement du colonial (un post-), une simple continuation. La rupture avec le passé introduite par le XIX ${ }^{\mathrm{e}}$ siècle est donc censée constituer la substance même de tout ce qui a suivi. Davantage que le XIX ${ }^{\mathrm{e}}$ siècle, je dirais donc que c'est l'histoire du colonialisme en Inde - même si elle acquiert au XIX $\mathrm{X}^{\mathrm{e}}$ siècle la plupart de ses caractéristiques - qui est vue comme l'unité historique clairement nouvelle et inclusive.

Bien sûr, le doute n'est pas permis quant à l'importance historique décisive de l'expérience coloniale et quant à son impact sur de nombreux aspects de la période post-coloniale. En même temps, ce genre d'approche de la temporalité colonisée masque de vigoureux éléments de continuité avec les passés pré-coloniaux ainsi que les mutations majeures survenues durant la période coloniale. Elle dissimule aussi, en partie, les éléments nouveaux qui permirent certaines évolutions post-coloniales. L'une des raisons en est évidemment que les archives indiennes n'autorisent pas les historiens à accéder aux documents post-coloniaux : l'histoire semble s'arrêter en 1947, avec l'Indépendance et la Partition, autrement dit, avec la fin de l'ère coloniale ${ }^{4}$. L'absence de sources historiques "normales» rend donc impossible d'écrire

3. Entre autres, Cf. Heike Liebau and al. (eds), The World in Wars. Experiences, Perspectives and Perceptions from the South, Leyde, Brill Book Series on Studies in Global Social History, 2010; Radhika Singha, "The Great War and A Proper Passport for the Colony: Border Crossing in British India, c. 1882-1922”, Indian Economic and Social History Review, n 3, 2013, p. 261-88.

4. Les archives indiennes suivent la règle des trente ans : officiellement, les lecteurs ne peuvent consulter que des documents âgés d'au moins trente ans. Dans la pratique, cependant, il est à peu près impossible d'obtenir les documents de la période post-indépendance. 
une histoire réellement détaillée des temps plus récents, et cela empêche une évaluation correcte de ce qui a changé en 1947, à quel point, et pourquoi.

Les perspectives sont en train de se transformer lentement. Depuis peu, certains chercheurs parlent des débuts d'une période moderne autonome qui dure du XVI ${ }^{e}$ au XVIII ${ }^{e}$ siècle, sinon plus longtemps encore ${ }^{5}$. Ce pourrait être une façon plus productive de re-périodiser l'histoire. D'autres ont commencé à se focaliser sur l'entre-deux-guerres comme unité historique distincte au sein de la période coloniale, et d'autres encore voient l'après-Seconde Guerre mondiale comme une entité historique nouvelle, qui a façonné de manière décisive la politique post-coloniale.

Selon moi, décomposer la période coloniale en plus petites unités nous rend plus sensibles à des processus d'évolution qui, sans être retentissants ni catastrophiques, n'en sont pas moins essentiels à leur manière. Cela attire aussi notre attention sur la continuité des changements, grands ou petits. On ne peut considérer le XIX ${ }^{\mathrm{e}}$ siècle comme la somme de tous les développements historiques simplement parce que l'autorité coloniale et les influences culturelles occidentales se sont établies à cette époque. L'entre-deux-guerres a entraîné des changements spectaculaires dans la politique coloniale et anticoloniale, au sein des tendances économiques et des pratiques sociales. L'impact de la Seconde Guerre mondiale fut immédiat et dévastateur car le conflit toucha l'Inde de très près, suscitant des ruptures radicales dans les mesures prises par l'État comme dans la politique populaire. La décolonisation et la Partition en furent certainement accélérées. Par certains côtés, les décennies post-indépendance ont prolongé le cadre de gouvernance coloniale, avec de solides éléments de continuité et de changement. En même temps, elles doivent être considérées comme une époque historique autonome, car leur contexte changea énormément avec l'Indépendance, la partition et le suffrage universel adulte.

RH19 : Votre thèse, intitulée Bengal 1928-1934: The Politics of Protest (1981), participe du profond renouveau de l'historiographie indienne à la fin des années 1970 et au début des années 1980 (avec notamment la publication des premiers volumes de la série des Subaltern Studies). Pourriez-vous revenir sur le contexte de ce renouveau et sur ses principales contributions?

Tanika Sarkar : Oui, ma thèse fut en effet un des fruits de l'influence considérable qu'eurent les premières Subaltern Studies, même si des approches très similaires, étudiant l'histoire par le bas, étaient déjà apparues auparavant

5. Entre autres, $c f$. Sanjay Subrahmaniyam, "Hearing Voices: Vignettes of Early Modernity in South Asia, 1400-1750”, Daedalus, volume 127, n 3 1998, p. 75-104, et Rosalind O’Hanlon, "Contested Conjunctures: Brahman Communities and Early Modernity in India", The American Historical Review, volume 118, n 3, 2013, p. 765-87. 
et ont continué en dehors du collectif à l'initiative des Subaltern Studies. Le contexte historique de ce changement historiographique inclut de nombreux éléments, dont je ne peux mentionner que quelques-uns. Les années 1960 furent une période d'importants soulèvements populaires de toutes sortes dans le monde entier : le mouvement de mai 1968 à Paris et ses répliques en Europe; la Révolution culturelle en Chine que beaucoup de gens perçurent - à tort - comme un soulèvement populaire entièrement émancipatoire et authentiquement révolutionnaire; la guerre du Vietnam; les mouvements pour les droits civiques aux États-Unis, et bien d'autres événements du même genre.

Tous ces phénomènes furent suivis de près en Inde, créant un espoir nouveau, une foi en la possibilité transformatrice de la politique subalterne, promesse qui parut confirmée dans notre pays par les premières années de la rébellion naxalite ${ }^{6}$, avec l'arrivée au pouvoir des partis de gauche dans quelques États, et avec la fin de la période répressive liée à l'état d'urgence, quand les droits démocratiques suspendus furent reconquis par le vote populaire, par les voix des individus subordonnés et marginalisés qui constituent la masse de l'électorat en Inde. Ce fut aussi une époque où commencèrent à s'effondrer des espoirs antérieurs, qui pensaient voir l'État indien postindépendance transformer l'héritage colonial en un ordre émancipatoire et équitable. Il semblait donc nécessaire de se détourner de l'étude des grands dirigeants pour s'intéresser aux masses subordonnées.

Le contexte historiographique fut créé par la tradition de l'histoire par le bas, en particulier les travaux de Christopher Hill, Eric Hobsbawm et peutêtre surtout d'E.P. Thompson : tous trois, et principalement ce dernier, ont créé une impulsion décisive pour approfondir la tradition préexistante d'histoires marxistes de la politique syndicaliste et de la participation paysanne aux mouvements anticoloniaux. Hill s'était rendu plusieurs fois en Inde et ses écrits étaient très lus par les historiens indiens. Ce furent pour eux des ressources extrêmement importantes afin de repenser l'histoire. La visite de Thompson à Delhi en 1977 eut un impact énorme car il s'est adressé à toute une génération de jeunes historiens, à l'université de Delhi et à l'université Jawaharlal Nehru. Les échanges qu'il eut avec eux à propos de ses travaux eurent une profonde influence sur eux, ainsi que son livre La formation de la classe ouvrière anglaise. Avec Sumit Sarkar, je lui ai rendu visite en Angleterre peu avant qu'il parte pour l'Inde, et de nombreuses fois par la suite. Les conversations que j'eus avec lui sur l'histoire et la politique furent aussi stimulantes que ses ouvrages.

Surtout, ce nouveau virage répudiait catégoriquement le mode nationaliste antérieur qui célébrait l'activisme populaire des mouvements nationaux

6. NdR : Ce mouvement naquit à la fin des années 1960 dans des zones tribales extrêmement pauvres du Bengale du nord, dans une ville appelée Naxalbari. Il était inspiré par les idéaux maoïstes d'insurrection rurale. Plus tard, il se propagea dans d'autres États. 
mais l'attribuait presque exclusivement aux qualités de meneur des grandes figures du nationalisme, tous issus de l'élite. Les historiens nationalistes ne reconnaissaient aucune autonomie aux combats subalternes, et ils n'étudiaient pas les mouvements subalternes qui se déroulèrent en parallèle avec les luttes nationalistes.

Dans les années 1960 et 1970, un groupe d'historiens, qui travaillaient à l'origine à l'université de Cambridge, rompirent tout à fait avec l'héroïsme et l'idéalisme des grands leaders indiens. S'appuyant sur les documents privés plutôt que sur les écrits et discours publics et publiés, ils montrèrent combien leur message différait de leurs véritables activités en coulisses. Dénonçant le nationalisme aussi bien que l'impérialisme, comme des inventions dénuées de toute substance, ils affirmaient que les unités-clés de l'histoire indienne moderne étaient les élites régionales et les relations de clientélisme locales, qui prirent l'ampleur de mobilisations nationales afin de tirer le maximum des contraintes, problèmes et opportunités liés à l'administration britannique. Ces historiens faisaient un usage intensif des archives et se concentraient sur le profil social des régions et des localités, sur les castes en particulier. Néanmoins, en rejetant à la fois l'impérialisme et le nationalisme comme des non-acteurs, ils allaient trop loin, et ils ignoraient complètement le rôle de l'idéologie et de mouvements anticoloniaux de masse qui comptent parmi les plus importants de l'histoire mondiale. Ce type de travaux historiques fut violemment condamné par la gauche nationaliste. Les Subaltern Studies furent la réaction la plus substantielle à l'approche de «l'école de Cambridge».

Dans sa première phase, le collectif des Subaltern Studies se focalisa sur la façon dont la population ordinaire - en particulier les paysans et la population tribale/Adivasi - interprétait les messages des dirigeants à sa propre manière et avec sa propre grille culturelle. Il éclairait aussi la manière dont cette population avait créé ses propres mouvements à l'intérieur des vastes mouvements nationalistes menés par l'élite, parfois même en bravant les directives de l'élite. Il introduisit aussi la notion de processus d'inversion dans la politique anticoloniale : c'est précisément aux moments où la répression étatique fut particulièrement intense, et où les leaders furent écartés des lieux d'action par le biais de l'emprisonnement, qu'apparut une politique subalterne formée et dirigée sans l'aide de l'élite, proposant des moyens entièrement neufs pour combattre les autorités coloniales. L'inverse se produisait aussi, quand les dirigeants maitrisaient pleinement les mouvements et pouvaient canaliser les initiatives subalternes dans la direction de leur choix. Ce fut un nouveau départ, très enthousiasmant, pour notre perspective historique. Cela permit aussi de s'intéresser de plus près aux cultures et aux mondes subalternes pour expliquer le militantisme politique des gens ordinaires.

Profondément impressionnée par cette perspective, j'ai tenté d'imiter dans mon propre travail certaines de ces nouvelles pistes. Mais alors que les subal- 
ternistes se concentraient majoritairement sur les luttes anticoloniales, j'ai voulu appliquer leur méthode à d'autres aspects, d'autres mouvements nés des luttes de pouvoir au sein de la société indienne : mouvements ouvriers, soulèvement contre les usuriers, mouvement communiste. J'ai étudié une brève période d'intense politique anticoloniale - de 1928 à 1934 - en tant qu'époque d'intersection de multiples tendances politiques au Bengale. Ces dernières années, je suis revenue sur certains de ces thèmes, mais avec une approche historique plus large, en incluant les combats et les grèves, mais en allant aussi au-delà.

Ce moment historiographique particulier fut très important parce qu'il se concentrait sur les événements spécifiques, régionaux et locaux, en termes d'initiatives politiques subalternes. Cette approche contrecarrait une perspective nationaliste antérieure, de mouvements anticoloniaux indifférenciés, où la population ordinaire exécutait, héroïque mais docile, les ordres de dirigeants issus de la classe moyenne et des castes supérieures. Par certains côtés, la perspective de l'école de Cambridge apporta un correctif utile à la vision monolithique et assez hagiographique des temps modernes qu'offraient les nationalistes, mais elle ignorait des événements d'importance capitale dans les mouvements de masse et ne pouvait donc pas apporter grand-chose à l'histoire du nationalisme anticolonial.

De leur côté, des historiens marxistes comme Ranajot Dasgupta et Sukomol Sen choisirent d'étudier un domaine du militantisme populaire qu'avaient négligé tant l'école de Cambridge que les nationalistes : la politique de la classe ouvrière, avec un intérêt particulier pour les syndicats, les grèves et le parti communiste qui en fut principalement responsable. Mais ils ignoraient les sphères de la caste et du genre, ainsi que les processus culturels à l'œuvre parmi les groupes subalternes qu'ils étudiaient.

En montrant une relation inverse entre politique subalterne et politique nationaliste de l'élite, les historiens subalternistes et d'autres qui travaillaient dans la même optique transformèrent notre compréhension des mouvements anticoloniaux, désormais dégagés d'un cadre nationaliste extrêmement simpliste. En même temps, les historiens subalternistes avaient encore besoin d'aller au-delà des mouvements de masse et des soulèvements pour expliquer les dimensions plus quotidiennes des vies subalternes en temps ordinaire, après la fin des luttes colossales. Ils devaient aussi mettre l'accent sur les contradictions de caste et de genre au sein des classes subalternes et entre elles. Plus tard, à partir du début des années 1990, il y eut un changement de perspective lorsqu'ils se mirent à se focaliser sur la résistance discursive à la domination culturelle coloniale, surtout de la part de la classe moyenne.

RH19 : Votre travail s'est orienté vers l'analyse des relations entre genre et construction des cultures politiques. Comment les gender studies sont-elles apparues dans le monde universitaire indien? Comment y 
ont-elles été reçues? Quelle position occupent-elles aujourd'hui au sein de la recherche, et plus précisément dans les programmes universitaires?

Tanika Sarkar : En ce qui concerne mon propre travail, je ne parlerais pas d'une orientation nouvelle. Dès mon premier livre, j'ai inclus tout ce que j'ai pu trouver sur le rôle des femmes dans les mouvements politiques que j'étudiais. En fait, je fus probablement l'auteure de la première monographie sur la politique nationaliste où, de manière limitée et hésitante, une place était faite au militantisme des femmes et aux relations genrées dans l'histoire du nationalisme. Bien sûr, il ne s'agissait pas d'une étude intensive du genre, et elle ne portait pas de façon exclusive ni même substantielle sur ce point, car je cherchais à aborder en même temps - mais bien trop légèrement, je le crains - plusieurs sphères politiques significatives.

Je me suis ensuite intéressée à des aspects spécifiques de la culture politique et de la vie sociale au XIX ${ }^{\mathrm{e}}$ siècle, en particulier à l'interface entre foi et droit. La fin des années 1980 et les années 1990 furent une époque d'essor considérable pour l'extrême droite hindouiste dans notre pays, et plusieurs d'entre nous se mirent à explorer simultanément des aspects de ce phénomène politique contemporain. J'ai ainsi tourné mon attention vers les racines du nationalisme culturel hindou, ou "renouveau hindou» qui remonte à la fin du $\mathrm{XIX}^{\mathrm{e}}$ siècle, et qui soutient l'idée que l'Inde est avant tout une nation d'hindouistes qui doivent empêcher les cultures et communautés non-hindouistes de s'affirmer. Cela impliquait surtout une résistance aux changements induits par le libéralisme moderne, dépeints comme le fruit de la domination coloniale étrangère. Bien sûr, en matière de nationalisme hindou, il existe des différences cruciales entre la fin du XIX ${ }^{e}$ et la fin $\mathrm{du} \mathrm{XX}^{\mathrm{e}}$ siècle, mais en travaillant à la fois sur deux fronts, pour ainsi dire, j'en suis venue à me demander pourquoi le Bengale, un des principaux sites modernes de réforme libérale, s'était par la suite tourné aussi résolument vers la défense des traditions hindoues. Beaucoup d'historiens très influents comme Partha Chatterjee et Dipesh Chakravarty ont travaillé sur le Bengale sans aborder cette question précise.

$\mathrm{Au} \mathrm{XIX}^{\mathrm{e}}$ siècle, le genre fut inévitablement un prisme important pour réimaginer et reconstituer les vies, les cultures et les nations. Par exemple, le nationalisme culturel du XIX ${ }^{\mathrm{e}}$ siècle se souciait surtout de pratiques matrimoniales hindoues et d'images hindoues de la vie conjugale : j'ai commencé à étudier ces discours et la culture politique entourant ces thèmes. Bien sûr, $j$ 'ai aussi travaillé sur le militantisme des femmes dans les domaines de la foi et de l'écriture. Au début des années 1990, une histoire féministe était apparue dans le milieu universitaire indien, qui poussa vers de nouvelles directions mon propre intérêt pour le genre. En même temps, je pense que j'ai toujours voulu étudier une période particulière, mais en me concentrant spécialement sur certains aspects, qui pouvaient aller du genre aux différentes politiques 
nationalistes, en passant par le droit et la littérature. Dans mon dernier livre, j'ai consacré plusieurs chapitres aux règles du veuvage hindou. Mais j'ai aussi étudié le théâtre public à Calcutta, les luttes des Adivasi au Bengale, la littérature politique et les livres pour enfants au Bengale aux $\mathrm{XIX}^{\mathrm{e}}$ et $\mathrm{XX}^{\mathrm{e}}$ siècles.

Il est impossible d'étudier une dimension de l'histoire sans comprendre l'évolution des relations genrées. En même temps, il est également impossible, je pense, de créer un compartiment étanche pour le genre comme catégorie à part, sans lien avec bien d'autres processus historiques simultanés.

L'histoire féministe systématique des relations genrées est née vers 1989, avec la publication de deux titres majeurs : Recasting Women, ouvrage collectif dirigé par Sangari et Vaid ${ }^{7}$, et Women in Colonial India, dirigé par J. Krishnamurty ${ }^{8}$. Si le premier adoptait un angle féministe marxiste pour examiner le patriarcat moderne sans doute introduit en Inde par le capitalisme colonial, le second n'avait pas de cadre fixé et reflétait des histoires multiples et parfois non commensurables à l'intérieur des questions de genres en Inde moderne. Le premier offrait aux historiens féministes un cadre conceptuel utilisable et multifonctions, mettant fortement l'accent sur la construction discursive du genre, mais le second ouvrait de nombreux thèmes historiques nouveaux où les distinctions genrées étaient cruciales - parmi la main-d'œuvre agricole et les ouvriers d'usine, par exemple, ainsi que dans diverses branches de la politique moderne.

Dans l'ensemble, les premières historiennes féministes indiennes furent des femmes de gauche, laïques et intéressées par les mouvements pacifistes, la politique anti-communautaire et les luttes pour les droits civiques (quand elles n'y étaient pas activement impliquées). Il leur fallut du temps pour obtenir la reconnaissance universitaire à l'intérieur de leur discipline. Les Women's Studies Centers finirent pourtant par avoir pignon sur rue et par devenir une composante familière des universités indiennes. L'historiographie féministe n'est plus une curiosité, elle n'est plus perçue comme incompatible avec une recherche ou un enseignement sérieux. La plupart des départements d'histoire proposent aussi quelques cours sur les femmes, surtout dans les programmes consacrés à l'histoire indienne moderne. Cependant, leur existence en tant que cours distincts dispense souvent les autres de devoir insérer l'histoire du genre dans le thème qu'ils abordent. Le genre reste donc un peu en dehors de l'histoire "conventionnelle». Partant, il existe une tendance à faire du genre un cours autonome ou un sujet de recherche à part, en partie déconnecté des autres évolutions historiques, un domaine autosuffisant, qui ne dérive pas des disciplines principales mais en interaction interdisciplinaire avec d'autres sujets, qui se focalise toujours entièrement sur le genre ou

7. Kumkum Sangari and Sudesh Vaid (eds), Recasting Women: Essays in Colonial History, Delhi, Kali for Women, 1989.

8. Jayasankar Krishnamurty (ed.), Women in Colonial India: Essays on Survival, Work and the State, Delhi, Oxford University Press, 1989. 
sur les femmes. Je pense que cette évolution ouvre d'immenses possibilités d'expansion à l'histoire des femmes et du genre. En même temps, elle a aussi quelque chose de limitatif pour les raisons exposées plus haut.

Dans la situation politique actuelle, ces centres courent le risque de devoir renoncer à leur vision politique critique pour se plier aux directives de l'État. Le gouvernement exige que cette discipline serve à inculquer la fierté nationale, surtout par rapport à un héritage hindou imaginaire. Je prévois donc pour l'avenir une bataille longue et difficile.

\section{RH19 : Parmi les jeunes historiens travaillant en Inde dans le domaine des gender studies, quels sont aujourd'hui les thèmes, les problèmes et les méthodes les plus en vogue?}

Tanika Sarkar : Les études de genre continuent à s'épanouir surtout en histoire moderne, même s'il existe quelques exceptions notables comme Uma Chakravarty, Kumkum Roy et d'autres qui ont travaillé sur l'Inde ancienne. Dans les années 1990, la recherche féministe fut fortement influencée par les perspectives post-coloniales qui, dans les universités américaines, étaient particulièrement bien établies dans les études littéraires post-coloniales et dans les centres d'étude de l'Asie du sud. L'accent était mis avant tout sur les discours coloniaux et occidentaux issus des Lumières, qui redéfinissaient les Indiennes selon un modèle patriarcal moderne et imposaient le silence à leur voix authentique au nom de valeurs libérales universelles. La modernité en Inde était considérée comme une phase sombre pour les femmes, ce qui empêchait pratiquement d'expliquer l'apparition du féminisme en Inde autrement que comme un mouvement d'imitation, pâle copie du féminisme occidental. De plus, le discursif était préféré à la description détaillée des évolutions économiques, des formes de travail et des mouvements politiques. Il y a là un paradoxe : alors que nous disions tous que ce qui était personnel relevait du politique, le domaine formel du politique même était oublié ou perçu comme une autre caractéristique de la modernité, où les femmes n'étaient entrées que pour subir de nouvelles formes de subordination. On s'est relativement peu intéressé aux normes orthodoxes et aux pratiques religieuses qui continuèrent largement à dominer l'existence des femmes. L'accent était plutôt mis sur les effets de l'État colonial et des réformateurs libéraux : en cherchant à réorganiser la vie du pays sur un modèle occidental moderne, ils avaient agi sur le vécu des femmes et avaient parfois supprimé les libertés et les adaptabilités coutumières.

Plus récemment, les histoires du genre ont élargi leurs horizons. On se focalise depuis peu sur les formes de travail genrées et sur la vie politique des femmes, avec notamment une reconceptualisation genrée de l'ensemble des mouvements politiques. De plus, de nouveaux domaines sont apparus au sein des études de genre, avec une insistance relativement nouvelle sur les 
études de la sexualité : travailleurs du sexe, communautés transgenre, désirs non-hétérosexuels, construction de la masculinité. L'émergence d'histoires des Dalits ou intouchables a favorisé les études situées à l'intersection de la caste et du genre, tant pour les "hautes castes» que pour les «basses castes"?. De nouvelles formes de créativité féminine sont explorées plus méthodiquement, dans les arts et l'artisanat, la musique, la religion et les pratiques littéraires.

RH19 : La question de l'enseignement de l'histoire - et notamment de l'histoire nationale - a suscité et continue de susciter en France de nombreux débats, entre les tenants du «roman national» et les partisans d'une perspective critique. Des débats de ce type, notamment sur le contenu des manuels scolaires, ont aussi lieu en Inde. Vous avez participé à l'écriture de certains manuels, pourriez-vous revenir par conséquent sur cette controverse? Et plus globalement sur les enjeux de l'enseignement de l'histoire en Inde aujourd'hui?

Tanika Sarkar : C'est une question très importante et très décriée, dont j'ai déjà évoqué certains éléments quand j'ai présenté l'histoire nationaliste des mouvements nationalistes. Tournons-nous maintenant vers les manuels et leur histoire récente en Inde. C'est une composante très intéressante de l'historiographie indienne. Beaucoup de nos principaux historiens ont participé à la rédaction de manuels pour les collèges, lycées et universités: Romila Thapar, Satis Chandra, Bipan Chandra, Sumit Sarkar, Sekhar Bandyopadhyay, Barbara and Thomas Metcalf, Ayesha Jalal et Sugata Bose, Ishita Bannerjee-Dube, parmi tant d'autres.

Initialement, un groupe d'éminents historiens appartenant à la gauche nationaliste ont voulu présenter aux écoliers une perspective résistant aux explications impérialistes occidentales comme aux interprétations de la droite indienne. Ils rédigèrent des ouvrages laïques et libéraux, introduisant les concepts de modes de production dans un langage simple et adapté. Cet ensemble de manuels resta en vigueur du début des années 1970 jusqu'à la fin des années 1990, avec le parrainage du Conseil national de la formation à l'éducation et à la recherche (NCERT). Toutes les écoles n'acceptaient pas ces manuels, mais ils étaient certainement utilisés très largement, puisqu'ils étaient l'œuvre d'historiens bien connus du pays.

En 1998, quand arriva au pouvoir un gouvernement dominé par la droite hindouiste, ces manuels furent remplacés par un ensemble entièrement neuf qui reflétait une orientation idéologique particulière : glorification de l'héri-

9. Sumit Sarkar and Tanika Sarkar (eds), Caste in Modern India, Ranikhet, Permanent Black, 2014; Gail Omvedt, Dalits and the Democratic Revolution, Delhi, Sage, 1994; Anupama Rao, The Caste Question, Ranikhet, Permanent Black, 2009; Pandian M. S. S., Brahman and Non Brahman: Genealogies of the Tamil Political Present, Ranikhet, Permanent Black, 2007, parmi bien d'autres. 
tage hindou, minimisation de l'histoire culturelle et politique non-hindoue, mise en avant du nationalisme hindou. Ces manuels furent rédigés par des historiens qui n'étaient ni établis ni connus dans le domaine de la recherche historique et, très vite, des distorsions et des erreurs grossières, dans les faits comme dans l'interprétation, furent relevées par un grand nombre de spécialistes. En 2004, ce gouvernement fut chassé du pouvoir, et le NCERT se mit à réfléchir à une nouvelle réforme des programmes et des manuels.

Il fut finalement décidé qu'au lieu de revenir aux anciens manuels, on en rédigerait de nouveaux, pour intégrer les dernières évolutions de la discipline historique dans l'ensemble de la planète, et pour faire de l'histoire un sujet intéressant et attirant pour les jeunes sur le plan pédagogique. Quantité de documents visuels furent ajoutés aux textes, les pages furent composées de manière à captiver l'attention de l'élève et à la retenir, et de nouveaux thèmes furent introduits, en résonance avec les expériences et intérêts de l'élève : le sport, par exemple. Au lieu de se focaliser sur le nationalisme comme dans les manuels de la gauche nationaliste, bien d'autres domaines de la recherche historique furent sollicités pour élargir l'horizon intellectuel : politique subalterne, mouvements de caste, culture matérielle, arts du spectacle et audiovisuel, par exemple. Au lieu de se voir proposer une posture d'autocélébration, les élèves étaient encouragés à examiner les problèmes et les abus à l'intérieur des traditions indiennes. Dans ces manuels, on examinait de près les sociétés non-occidentales autant qu'occidentales, ainsi que l'histoire sociale et culturelle de nombreux pays du monde. Surtout, les élèves étaient incités à se montrer critiques, à penser par eux-mêmes, à relier des domaines jusque-là maintenus séparés. Les manuels furent préparés par de longues conversations et par l'apport actif des enseignants.

Le gouvernement vient à nouveau de changer, la droite hindouiste est revenue au pouvoir, cette fois, avec une majorité écrasante. Les organismes et groupes de réflexion de droite ont déjà exigé du NCERT des explications sur de nombreux points concernant les derniers manuels. Bien que les institutions de recherche en sciences sociales aient été entièrement reprises en mains par des adeptes d'une idéologie de droite, les manuels du NCERT sont encore en usage. C'est étonnant, mais ce n'est guère rassurant. Échaudé par les erreurs contenues dans ses précédents manuels, le gouvernement se donne cette fois le temps d'en préparer de nouveaux, avec une révision plus systématique et approfondie du sens même de l'histoire. Le décor a d'abord été planté par la nomination d'hommes - j'utilise le mot "hommes» à dessein - de leur couleur politique à la tête des institutions de recherche. Ils choisiront avec soin comment écrire les nouveaux manuels.

Néanmoins, les signes sont déjà manifestes; permettez-moi de citer quelques exemples. Dans un texte récent, Swapan Dasgupta, l'un des principaux idéologues de la droite hindouiste, a formulé une déclaration qui pourrait surprendre au premier abord. Il a dit que s'il était entièrement permis 
aux historiens occidentaux de célébrer l'histoire de leurs empires, les historiens indiens devaient pouvoir célébrer les mouvements nationalistes contre l'autorité étrangère. Cela va bien au-delà d'un relativisme extrême, et revient à dire que l'histoire n'est pas importante en soi, mais n'est qu'un instrument qui peut être modelé en fonction d'intérêts politiques divers, suivant les besoins du nationalisme. La même histoire peut être ainsi présentée selon des modes totalement différents dans des contextes radicalement différents. Cette démarche dénie évidemment la valeur intrinsèque de la discipline comme système de connaissance. Elle en fait un instrument idéologique particulier, au service de la glorification nationale, où les diversités n'entrent pas en contact les unes avec les autres, mais restent compartimentées comme des constructions autosuffisantes.

Ces derniers temps, l'Histoire est peut-être le champ de savoir le plus controversé, alors que la droite hindouiste justifie ses revendications politiques en premier lieu par des constructions historiques. Elle affirme ainsi que les Hindous étaient jadis un grand peuple glorieux et parfait, excellant dans tous les domaines possibles, du militaire au scientifique; que les invasions musulmanes, par leur cruauté et leur caractère oppressif, les ont réduits à la subordination, en multipliant les conversions forcées et les agressions envers les femmes, avant de diviser le pays pour créer le Pakistan. De même, la droite hindouiste suggère que les musulmans conspirent encore pour islamiser l'Inde, en enlevant et convertissant des Hindoues et par des attaques terroristes; et que sous l'autorité britannique, les esprits hindous furent corrompus par des valeurs et des normes socioculturelles occidentales. Cette Histoire-là est chargée d'un message clair et sonore : tous les torts passés doivent être vengés dans le présent. Du fait du contenu de leur politique, l'Histoire est, de manière évidente, l'outil le plus important.

Les ministres et les conseillers intellectuels ont expliqué que l'Histoire devait être l'étude de l'héritage hindou, et viser à inculquer les gloires nationales. Tout ce qui ressemble de près ou de loin à une critique ou à une réflexion, tout ce qui signale aux élèves les notions d'injustice sociale dans les castes, les genres ou les populations tribales, tout ce qui attire l'attention sur l'histoire indienne non-hindoue, tout ce qui détourne de la glorification de la nation hindoue doit être éliminé comme antinational et séditieux. Au lieu que l'Histoire englobe tout ce qui se passe dans le monde - de sorte que «rien de ce qui est humain ne m'est étranger» - il ne faut livrer aux élèves que des «faits» d'un certain type : les faits construits à partir des épopées, des mythes et des rumeurs hindous. Les procédures savantes ordinaires, d'authentification des sources, n'ont absolument aucune pertinence. L'accent doit aussi être mis sur l'Inde seule, en ignorant le reste du monde : il a été reproché aux derniers manuels du NCERT de beaucoup trop exposer les élèves à l'histoire non-indienne, et il a été dit que l'histoire des révolutions - de la Révolution française en particulier - pouvait sans problème être exclue des programmes. 
Dans l'ensemble des questions posées sur un de mes chapitres consacrés à la réforme sociale moderne, par exemple, j'ai été accusée de semer le trouble en parlant longuement d'E. V. Ramaswamy Naicker, un Indien du sud, athée, qui, au début du XX $X^{\text {e }}$ siècle, se livra à une critique des relations de caste et de genre. On m'a aussi demandé pourquoi j'avais décrit Sayyad Ahmad Khan, modernisateur musulman de la fin du XIX ${ }^{e}$ siècle, comme un réformateur social. On m'a aussi reproché de mentionner Pandita Ramabai, qui a œuvré inlassablement pour améliorer la situation des veuves hindoues des castes supérieures, dans l'ouest de l'Inde à la fin du XIX $\mathrm{X}^{\mathrm{e}}$ siècle, sous prétexte qu'elle s'était convertie au christianisme et ne devait donc être vue que comme un agent de l'Empire et des missionnaires. Des questions de semblable nature ont été posées à la plupart des auteurs.

La droite hindouiste affirme que tout ce qui favorise le sentiment de la gloire nationale/hindoue est bon. Il y a donc un risque de voir les manuels se remplir d'inventions et de distorsions factuelles, sans donner aux élèves les outils pédagogiques leur permettant de s'assurer que ce qu'ils lisent a du sens ou peut être vrai, s'il est plausible par exemple que le dieu Ganesh à tête d'éléphant soit le fruit de l'antique chirurgie esthétique hindoue, pour s'en tenir à une revendication formulée au plus haut niveau politique.

Surtout, les élèves apprendront à craindre et à détester les non-hindous comme des agresseurs trompeurs, des individus de basse extraction greffés sur notre corps politique, qui ont fait des ravages parmi les hindous innocents. Et ils l'apprendront d'une manière qui leur inspirera un désir de revanche et les incitera à se joindre aux campagnes haineuses contre les musulmans, les chrétiens, le Pakistan, la gauche et les féministes.

Je ne sais pas vraiment comment l'impératif nationaliste a pu être introduit et développé dans les manuels français. Mais j'ai peine à croire que cela repose sur un mépris total pour les faits fondamentaux de l'Histoire. En ce sens, les futurs manuels indiens fixeront sans doute de nouvelles normes.

Propos recueillis par courriel par $V$. C. et $F$. $B$. en septembre et décembre 2018, et traduits de l'anglais par Laurent Bury. 